Real Analysis Exchange

Vol. 24(2), 1998/9, pp. 791-797

Mihaela Racilă, Department of Mathematics, University of Craiova, A. I.

Cuza 13, 1100 Craiova, Romania; e-mail: Mpred@udjmath1.sfos.ro

\title{
ON SOME PROPERTIES OF QUASI-UNIFORM CONVERGENCE AND QUASI-CONTINUOUS FUNCTIONS
}

\begin{abstract}
In this note some properties of the quasi-uniform convergence of [9] are studied and related to the quasi-continuous functions of [5] (in terms of the entourages [1]). Then, quasi-uniform convergence is investigated as a convergence structure in the sense of [6].

Also, quasi-uniform and quasi-normal convergences of sequences of real-valued functions from [2], [3] are compared.
\end{abstract}

\section{Introduction}

Trying to explain when a limit of a sequence of quasi-continuous functions is quasi-continuous, Irena Domnik [5] used a type of quasi-uniform convergence introduced by Maria Predoi in [9].

Because the uniform structure can be defined in terms of a family of pseudometrics on the space [6] (Theorem 20.11, 20.13) and quasi-continuity can be characterized with a real valued function of the oscillation type (Theorem $1,[5])$, in [5] one considered a family of pseudo-metrics to obtain results in uniform spaces.

We shall now present some of the major results in terms of the uniformity, where we have used entourages.

On the other hand, in [4] it is shown that the set of all real valued functions defined on topological space $X$ endowed with PrQU convergence is an $\mathcal{L}$-space (in the sense of [7]), but this is not true for any of the ArQU, GaQU and SPQU convergences.

We shall present and investigate PrQU convergence as a convergence structure in a more general situation. We will prove that the quasi-uniform convergence of [9] is a topological convergence in the sense of [6].

In the final part of the work we compare this with quasi-normal convergence introduced in [2],[3].

Mathematical Reviews subject classification: 26A15

Received by the editors January 8, 1998 


\section{Sequences of quasi-continuous functions}

At first we will present definitions which we will use.

Definition 2.1. [5] Let $X$ be topological space. A set $A \subset X$ is said to be semi-open if $A \subset \overline{I n t A}$. A set $A \subset X$ is called semi-neighbourhood of a point $x_{0} \in X$ if $A$ is semi-open set and $x_{0} \in A$.

Definition 2.2. Let $X$ be a topological space and let $Y$ be a uniform space with a family $\mathcal{P}$ of pseudo-metrics. A function $f: X \rightarrow Y$ is said to be quasicontinuous at a point $x_{0} \in X$ if for every $\varepsilon>0$ and every pseudo-metric $d \in \mathcal{P}$ there exists a semi-neighbourhood $C$ of the point $x_{0}$ such that, for every point $x \in C$,

$$
d\left(f\left(x_{0}\right), f(x)\right)<\varepsilon
$$

We now state the following theorem relative to the quasi-continuity in terms of entourages ([1], pg. 169).

Theorem 2.1. Let $X$ be a topological space and let $(Y, \mathcal{U})$ be a uniform space. A function $f: X \rightarrow Y$ is quasi-continuous at a point $x_{0} \in X$ iff for every $U \in \mathcal{U}$ there exists a semi-neighbourhood $C$ of the point $x_{0}$ such that, for every $x \in C$,

$$
\left(f(x), f\left(x_{0}\right)\right) \in U
$$

Proof. For the uniformity $\mathcal{U}$ there exists an augmented associated family $\mathcal{P}$ of pseudo-metrics. The family $\{U(d, \varepsilon) ; d \in \mathcal{P}, \varepsilon>0\}$, where $U(d, \varepsilon)=$ $\{(x, y): d(x, y)<\varepsilon\}$ is a base for $\mathcal{U}$. (see [6],[8]) Let $U$ be an arbitrary element of $\mathcal{U}$. Then there exists $d \in \mathcal{P}$ and $\varepsilon>0$ such that $U \supset U(d, \varepsilon)$.

If the function $f$ is quasi-continuous at $x_{0} \in X$, according to Definition 2.2, the pair $d, \varepsilon$ determines a semi-neighbourhood $\mathrm{C}$ of the point $x_{0}$, such that, for every point $x \in C, d\left(f\left(x_{0}\right), f(x)\right)<\varepsilon$. This is equivalent to $\left(f\left(x_{0}\right), f(x)\right) \in$ $U(d, \varepsilon)$ hence $\left(f\left(x_{0}\right), f(x)\right) \in U$ for every point $x \in C$. Thus quasi-continuity implies the condition of the theorem.

If the condition of the theorem is satisfied, for $\varepsilon>0$ and $d \in \mathcal{P}$, there exists a semi-neighbourhood $\mathrm{C}$ of the point $x_{0}$ such that

$$
\left(f\left(x_{0}\right), f(x)\right) \in U(d, \varepsilon), \quad \text { for every } \quad x \in C,
$$

because $U(d, \varepsilon) \in \mathcal{U}$. This means that $d\left(f\left(x_{0}\right), f(x)\right)<\varepsilon$, for every $x \in C$, so that $f$ is quasi-continuous at $x_{0}$.

With the help of the preceding theorem we show now that the limit of a quasi-uniformly convergent net of quasi-continuous functions is quasicontinuous. 
Definition 2.3. [9] Let $\mathcal{F}$ be the set of all functions defined on topological space $X$ with the values in the uniform space $(Y, \mathcal{U})$. Let $(A, \leq)$ be a directed set, $\pi: A \rightarrow \mathcal{F}$ a net of functions, $\pi(a)=f_{a}$. The net $\pi$ is called quasiuniformly convergent to the function $f: X \rightarrow Y$ if for every point $x_{0} \in X$ and $U \in \mathcal{U}$ there exists $a_{0} \in A$ such that for every $a \in A, a_{0} \leq a$, there is a neighbourhood $V$ of the point $x_{0}$ with the property $\left(f_{a}(x), f(x)\right) \in U$, for every point $x \in V$.

Theorem 2.2. If the net $\pi$ of quasi-continuous functions is quasi-uniformly convergent to a function $f$, then $f$ is quasi-continuous.

Proof. Let $x_{0} \in X$ and $U \in \mathcal{U}$. Let $U^{\prime} \in \mathcal{U}$ be a symmetric entourage such that $U^{\prime} \circ U^{\prime} \circ U^{\prime} \subset U$. Since the net $\pi$ quasi-uniformly converges to the function $f$, for $U^{\prime}$ there exists $a_{0} \in A$ such that for every $a \in A, a_{0} \leq a$, we can find the neighbourhood $V$ of the point $x_{0}$ with the property:

$$
\left(f_{a}(x), f(x)\right) \in U^{\prime}, \text { for every point } x \in V .
$$

As a consequence,

$$
\left(f_{a}\left(x_{0}\right), f\left(x_{0}\right)\right) \in U^{\prime} .
$$

Let $a \in A, a_{0} \leq a$ fixed. From the assumption the function $f_{a}$ is quasicontinuous at the point $x_{0}$, so for $U^{\prime}$ there exists a semi-neighbourhood $C^{\prime}$ of the point $x_{0}$ with the property:

$$
\left(f_{a}(x), f_{a}\left(x_{0}\right)\right) \in U^{\prime}, \text { for all points } x \in C^{\prime} .
$$

If $C=V \cap C^{\prime}$, obviously, $\mathrm{C}$ is a semi-neighbourhood of the point $x_{0}$ and according to (1), (2), (3), for every $x \in C$, we have:

$$
\left(f(x), f\left(x_{0}\right)\right) \in U^{\prime} \circ U^{\prime} \circ U^{\prime} \subset U
$$

As a consequence, the condition $\left(f(x), f\left(x_{0}\right)\right) \in U$, for every $x \in C$ is satisfied.

According to the above theorem, this means that the function $f$ is quasicontinuous at the point $x_{0}$ and the proof is completed.

Remark 2.1. From the above theorem we can deduce Theorem 2 and Corollary 3 of [5]. 


\section{Some convergence structure}

We will prove now that the quasi-uniform convergence from [9] is equivalent to a topological structure in the sense of [6].

Theorem 3.1. The quasi-uniform convergence on $\mathcal{F}$ (introduced by definition 2.3) is a topological convergence, i.e. it satisfies the following conditions:

$\left[c_{1}\right]$ For each $f \in \mathcal{F}$ and for each directed set $(A, \leq)$, if $\pi: A \rightarrow\{f\}$ then $\pi$ converge quasi-uniformly to the function $f$;

[c․ If $\pi: A \rightarrow \mathcal{F}$ is a net which converge quasi-uniformly to the function $f \in \mathcal{F}$, then each subnet of $\pi$ converge quasi-uniformly to $f$;

[c] If $\pi: A \rightarrow \mathcal{F}$ is a net which is not quasi-uniformly convergent to $f \in \mathcal{F}$, then there exists a subnet of $\pi$ such that none of its subnets converges quasi-uniformly to $f$.

[c $\left.c_{4}\right]$ Let $\pi: D \rightarrow \mathcal{F}, \pi(m)=f_{m}$, be a net which converges quasi-uniformly to $f \in \mathcal{F}$. For every $m \in D$, let $\pi_{m}: E_{m} \rightarrow \mathcal{F}, \pi_{m}(n)=f_{m n}$, be a net which converges quasi-uniformly to $f_{m}$. If $\widetilde{D}=D \times \underset{m \in D}{\times} E_{m}$ is endowed with the product order and $\widetilde{\pi}: \widetilde{D} \rightarrow \mathcal{F}$ is the diagonal subnet (in the sense of [6]) defined by

$$
\widetilde{\pi}(m, \varphi)=\pi_{m}(\varphi(m))=f_{m \varphi(m)},
$$

then $\widetilde{\pi}$ converges quasi-uniformly to $f$.

Proof.

$\left[c_{1}\right]$ is trivial.

$\left[c_{2}\right]$ Let $\pi: A \rightarrow \mathcal{F}, \pi(a)=f_{a}$, be a net which converges quasi-uniformly to $f \in \mathcal{F}$. Let $(B, \leq)$ be a directed set, $h: B \rightarrow A$ a Kelley's application and $\pi \circ h: B \rightarrow \mathcal{F}$ a subnet of $\pi$. We show that $\pi \circ h$ converges quasi-uniformly to $f \in \mathcal{F}$. Let $U \in \mathcal{U}$ and $x_{0} \in X$. Since $\pi$ converges quasi-uniformly to $f$, there exists $a_{0} \in A$ such that for every $a_{0} \in A, a_{0} \leq a$, one can find a neighbourhood $V_{a}$ of the point $x_{0}$ with the property

$$
\left(f_{a}(x), f(x)\right) \in U, \quad \text { for every } \quad x \in V_{a}
$$

But $h: B \rightarrow A$ is a Kelley's application, i.e. for any $a_{0} \in A$ (as before) there exists $b_{0} \in B$ such that $b_{0} \leq b$ implies $a_{0} \leq h(b)$. 
Now from $h(b) \in A$ and $a_{0} \leq h(b)$ it follows that there exists a neighbourhood $V_{h(b)}$ of the point $x_{0}$ such that

$$
\left(f_{h(b)}(x), f(x)\right) \in U, \quad \text { for every } \quad x \in V_{h(b)} .
$$

Hence the subnet $\pi \circ h$ converges quasi-uniformly to $f$.

$\left[c_{3}\right]$ We suppose that the net $\pi: A \rightarrow \mathcal{F}, \pi(a)=f_{a}$, is not quasiuniformly convergent to $f$. It follows that there exists $x_{1} \in X, U_{1} \in \mathcal{U}$ and a cofinal subset $A_{1} \subset A$ with the property that for every neighbourhood $V$ of the point $x_{1}$ there exists $\bar{x} \in V$ such that

$$
\left(f_{a_{1}}(\bar{x}), f(\bar{x})\right) \notin U_{1}, a_{1} \in A_{1} .
$$

Obviously, $\left\{f_{a}\right\}_{a \in A_{1}}$ is a subnet of $\pi$ with the property that each of its subnet is not quasi-uniformly convergent to $f$.

$\left[c_{4}\right]$ Let $U \in \mathcal{U}, x_{0} \in X$, be arbitrary and $U_{1} \in \mathcal{U}$ be a symmetric entourage such that $U_{1} \circ U_{1} \subset U$. Since the net $\pi$ is quasi-uniformly convergent to $f$, for $U_{1} \in \mathcal{U}$ and $x_{0} \in X$ there exists $m_{0} \in D$ such that for each $m \geq m_{0}$ there exists a neighbourhood $V_{m}^{\prime}$ of the point $x_{0}$ such that

$$
\left(f_{m}(x), f(x)\right) \in U_{1}, \quad \text { for every } \quad x \in V_{m}^{\prime} .
$$

For $m \geq m_{0}$, since $\pi_{m}$ is quasi-uniformly convergent to $f_{m}$, there exists $\varphi_{0}(m) \in E_{m}$ such that for each $n \in E_{m}, n \geq \varphi_{0}(m)$ there exists a neighbourhood $V_{n}$ of the point $x_{0}$ such that

$$
\left(f_{m n}(x), f_{m}(x)\right) \in U_{1}, \quad \text { for every } \quad x \in V_{n} .
$$

If $m \leq m_{0}$, let $\varphi_{0}(m) \in E_{m}$ be arbitrary. Therefore, $\left(m_{0}, \varphi_{0}\right) \in \widetilde{D}$. If $(m, \varphi) \geq\left(m_{0}, \varphi_{0}\right)$ it follows $m \geq m_{0}$ and $\varphi \geq \varphi_{0}$.

For $m \geq m_{0}$, the condition (4) is satisfied and $\varphi \geq \varphi_{0}$ implies $\varphi(m) \geq \varphi_{0}(m)$.

According to (5), there exists a neighbourhood $V_{m}^{\prime \prime}$ of the point $x_{0}$ such that

$$
\left(f_{m \varphi(m)}(x), f_{m}(x)\right) \in U_{1}, \quad \text { for every } \quad x \in V_{m}^{\prime \prime} .
$$

Then $V_{m}=V_{m}^{\prime} \cap V_{m}^{\prime \prime}$ is a neighbourhood of the point $x_{0}$ and for every $x \in V_{m}$, according to (4) and (6), we have

$$
\left(f_{m \varphi(m)}(x), f(x)\right) \in U_{1}, \circ U_{1} \subset U .
$$

This proves that $\widetilde{\pi}$ is quasi-uniformly convergent to $f$ and the proof is finished. 


\section{Quasi-uniform and quasi-normal convergences of se- quences of real-valued functions}

Definition 4.1. [2],[3] Let $f_{n}, f, n=0,1,2, \ldots$ be real-valued functions defined on a set $X$. We shall say that the sequence $\left\{f_{n}\right\}_{n \in N}$ converges quasinormally to $f$ on $X$, if there is a sequence $\left\{\varepsilon_{n}\right\}_{n=0}^{\infty}$ of non-negative reals converging to zero such that for every $x \in X$ there is an index $k_{x}$ such that

$$
\left|f_{n}(x)-f(x)\right| \leq \varepsilon_{n}
$$

for every $n \geq k_{x}$.

Remark 4.1. If $X$ is a topological space and $f_{n}, f, n=0,1,2, \ldots$ are real-valued functions defined on $X$, according to definition 2.3 , the sequence $\left\{f_{n}\right\}_{n \in N}$ converges quasi-uniformly to $f$ on $X$ if for every $x_{0} \in X$ and $\varepsilon>0$ there exists $n_{0} \in N$ such that for every $n \geq n_{0}$ there is a neighbourhood $V_{n}$ of the point $x_{0}$ with the property

$$
\left|f_{n}(x)-f(x)\right|<\varepsilon
$$

for every point $x \in V_{n}$.

Remark 4.2. According to [2],[9], the quasi-uniform convergence, but also the quasi-normal convergence imply pointwise convergence, and both are implied by the uniform convergence.

However, neither quasi-uniform convergence nor the quasi-normal convergence implies the other. Some simple examples show this.

Example 4.1. Let $f_{n}(x)=x^{n}$ for $x \in[0,1]$ and $f(x)=0$ for $x \in[0,1)$, $f(1)=1$. Then $\left\{f_{n}\right\}_{n \in N}$ converges quasi-normally to $f$ on $[0,1]$ but not quasi-uniformly.

Example 4.2. The sequence $\left\{g_{m}\right\}_{m \in N}$ of continuous functions on $\mathbf{R}$ from [2], example 1.7., converges pointwise to a continuous function (zero) on $\mathbf{R}$, but does not quasi-normally. According to [9], the sequence $\left\{g_{m}\right\}_{m \in N}$ converges quasi-uniformly to zero on $\mathbf{R}$.

Example 4.3. Let $f_{n}(x)=x^{n}$ and $f(x)=0$ for $x \in(0,1), n \in N$. Then $\left\{f_{n}\right\}_{n \in N}$ converges quasi-normally and quasi-uniformly to $f$ on $(0,1)$.

Example 4.4. The sequence $\left\{f_{n}\right\}_{n \in N}$ of continuous functions on $\mathbf{R}$ from [2], example 1.4., converges pointwise to $f$ on $\mathbf{R}$, but not quasi-normally. Since $f$ is not continuous, it follows that $\left\{f_{n}\right\}_{n \in N}$ does not converge quasi-uniformly. 
Quasi-Uniform Convergence And Quasi-Continuous Functions 797

Remark 4.3. If $X$ is a $Q N$-space in the sense of [3], then the quasi-uniform, quasi-normal and pointwise convergence coincide in $\mathcal{C}(X)$.

Further, in order to compare the quasi-uniform and quasi-normal convergence, we can easily see that these two coincide in $\mathcal{C}(X)$ if and only if $X$ is a QN-space.

\section{References}

[1] Bourbaki, Elements of Mathematics, General Topology, Part 1, Addison Wesley, London, 1966

[2] Bukovská Z., Quasi-normal convergence, Math. Slovaka 41 (2) (1991), 137146.

[3] Bukovsky L., Reclaw I. and Repicky M., Spaces not distinguishing pointwise and quasi-normal convergence of real functions, Topology Appl. 41 (1991), 25-40.

[4] Bukovská Z., Bukovsky L., Ewert J., Quasi-uniform convergence and $\mathcal{L}$ spaces, Real Anal. Exch., 18 (2), (1992-'93), 321-329.

[5] Domnik I., On some characterizations of quasi-continuous functions, Analele Univ. din Craiova, Seria Math.-Inf., XXI (1994), 12-20.

[6] Kelley, J.L., General topology, Van Nostrand, Toronto, New York, London, 1957.

[7] Kuratowski K., Topologie I, PWN, Warszawa, 1958.

[8] Murdershwar, M.G., Naimpally, S.A., Quasi-uniform topological spaces, Noordhoff, Amsterdam, 1966.

[9] Predoi M., Sur la convergence quasi-uniforme, Periodica Math. Hungarica, 10 (1), (1979), 31-40. 